\title{
La réintégration du travailleur dans l'entreprise après un jugement de première instance
}

\section{Anna Musiała}

\section{(2) OpenEdition}

1 Journals

\section{Édition électronique}

URL : https://journals.openedition.org/rdctss/1265

DOI : $10.4000 /$ rdctss. 1265

ISSN : 2262-9815

Éditeur

Centre de droit comparé du travail et de la sécurité sociale

\section{Édition imprimée}

Date de publication : 1 avril 2020

Pagination : 200-203

ISSN : 2117-4350

\section{Référence électronique}

Anna Musiała, «La réintégration du travailleur dans l'entreprise après un jugement de première instance », Revue de droit comparé du travail et de la sécurité sociale [En ligne], 1 | 2020, mis en ligne le 01 novembre 2021, consulté le 11 novembre 2021. URL : http://journals.openedition.org/rdctss/1265 ; DOI : https://doi.org/10.4000/rdctss. 1265

\section{cc)}

Revue de droit comparé du travail et de la sécurité sociale est mise à disposition selon les termes de la Licence Creative Commons Attribution - Pas d'Utilisation Commerciale - Pas de Modification 4.0 International. 


\section{ANNA MUSIALA}

Université de AdAM MiCKIEWICZ À POZNAŃ

\section{LA RÉINTÉGRATION DU TRAVAILLEUR DANS L'ENTREPRISE APRÈS UN JUGEMENT DE PREMIÈRE INSTANCE}

A l'automne 2019, une "grande réforme " du Code de procédure civile [Kodeks postępowania cywilnego, ci-après dénommé c.p.c.] est entrée en vigueur. Cette réforme compte parmi les modifications législatives les plus commentées de l'année écoulée, tant du point de vue de son ampleur (plus de 300 nouvelles dispositions), que de l'élargissement du contexte dans lequel ces modifications ont été introduites dans l'appareil judiciaire polonais. La nécessité d'accélérer la procédure a souvent été évoquée pour justifier son introduction (selon les données les plus récentes, la durée moyenne de la procédure judiciaire est d'environ 6 mois $^{1}$, mais en matière de droit du travail, elle se prolonge jusqu'à 18 mois environ).

Si les effets de cette réforme ne pourront être évalués qu'après un certain recul, on peut désormais affirmer qu'ils contribueront sensiblement au respect du droit des travailleurs dont le contrat de travail a été rompu de façon illégale.

L'une des principales dispositions fondamentale de la réforme concerne l'article 477 § 2 qui, jusqu'au 7 novembre 2019, énonçait : " Considérant la résiliation du contrat comme dépourvue d'effet, le tribunal, à la demande du salarié, peut dans son jugement imposer à l'entreprise le maintien de la relation de travail avec le salarié jusqu'au jugement définitif ». Cet article ne pouvait donc s'appliquer que dans les situations où le tribunal du travail prononçait un jugement avant la période de préavis. C'est également cet article qui justifie le projet de loi : " La pratique montre que les procès visant à obtenir la nullité de licenciement, et qui aboutissent dans un délai permettant d'appliquer l'art. 4772 \& , sont quasiment inexistants.

Afin de donner à cet article une réelle signification, il convient d'élargir les conditions de son application à la réintégration du salarié dans ses fonctions $»^{2}$.

La modification du c.p.c. a remplacé le libellé « lieu de travail/ entreprise » par " employeur », ce qui reflète effectivement d'un point de vue terminologique la portée subjective de l'obligation de prolonger la relation contractuelle et d'ajuster la durée de cette obligation. Mais le changement le plus important de cette disposition est de créer une obligation, lorsqu'il s'agit de la réintégration du salarié dans ses fonctions. La réintégration, autrement dit l'application des articles $45 \S 1$ et $56 \S 1$ du Code du travail est pertinente lorsque la résiliation du contrat de travail

1 https://isws.ms.gov.pl/pl/porownania-krajowe/

2 http://orka.sejm.gov.pl/Druki8ka.nsf/0/166CCC44490F3965C1258384003CD40A/\%24Fi le/3137-uzas.pdf 
est injustifiée ou viole les dispositions relatives à la rupture du contrat de travail, et que le tribunal du travail estime que la prise en compte d'une telle exigence n'est ni impossible, ni inutile.

Cette modification, votée grâce au lobby de "Solidarité " [NSZZ, Solidarność], le syndicat le plus important en Pologne, a été considérée comme l'une des plus polémiques ${ }^{4}$. De nombreux arguments furent avancés contre la réforme, principalement liés aux risques d'engendrer le tumulte dans l'organisation de l'entreprise et d'exposer l'employeur à des coûts supplémentaires. II est en effet possible que le tribunal de seconde instance annule le jugement du tribunal de première instance, obligeant ainsi l'employeur à maintenir le contrat de travail.

La doctrine démontre, à juste titre, qu'en l'état actuel, la loi autorise à imposer l'obligation de réintégration jusqu'à ce que la décision acquiert force de chose jugée, que ce soit dans le cas d'une rupture du contrat de travail avec ou sans préavis ${ }^{5}$. II convient ici d'attirer l'attention sur le fait que l'exécution de cette disposition revêt un caractère facultatif, et que le tribunal est censé considérer la demande du travailleur sans que celle-ci soit contraignante.

D'un autre côté, les arguments en faveur de cette modification avancent le fait que cette réforme traduit mieux l'intention du législateur que la version précédente de la disposition. Il semble en effet que l'intention du législateur était d'introduire dans la procédure civile un élément lié à la protection du travail, celui-ci étant considéré par le système judiciaire comme le moyen principal de subsistance des citoyens.

Le salarié étant une partie à la relation de travail - économiquement et socialement - plus faible que l'employeur, il est donc justifié de le privilégier juridiquement. Le droit du travail assume une fonction protectrice du droit, qui s'exprime particulièrement dans la protection de la vie et de la santé du travailleur, de ses intérêts matériels, ainsi que dans la règlementation du licenciement. La possibilité d'imposer des sanctions, aussi bien administratives que pénales, est également associée à cette fonction protectrice.

3 http://www.solidarnosc.org.pl/torun/ochrona-przed-bezprawnym-zwolnieniem/

4 https://www.infor.pl/prawo/praca/pracownik-przed-sadem/3056344,Obowiazekprzywrocenia-pracownika-do-pracy-moze-powodowac-wiecej-szkody-niz-pozytku.html; https://ksiegowosc-budzetowa.infor.pl/kadry-iplace/zatrudnianie-izwalnianie/3068563,Od-7-listopada-2019-r-sad-I-instancji-bedzie-mogl-nalozycobowiazek-dalszegozatrudnieniazwolnionego-pracownika.html; https://bezprawnik.pl/przywrocenie-pracownika-do-pracy/; https://serwisy.gazetaprawna.pl/praca-i-kariera/artykuly/1424810,zmiany-wzwolnieniach-z-pracy-i-przywrocenia-do-prancy.html

5 J. May, "Art. 477(2) Rigueur d'exécution immédiate : Accueil du recours et arrêt de maintien du contrat de travail», in T. Remburski, Les frais de justice en matière civile. Recours judiciaires collectifs. Dispositions transitoires. Commentaire sur les modifications, c.p.c., Tome I et II. 
Dans le cas de l'art. 477 \& 2 c.p.c., la question consiste à déterminer s'il est possible d'appliquer cette règle dans le cadre d'une procédure civile. Si l'on prend en compte le point de vue de la doctrine ${ }^{6}$ concernant le privilège du salarié, ainsi que le fait que la garantie pour le salarié de défendre ses droits en matière de droit du travail est identique à celle pratiquée dans la procédure civile, on aboutit à la conclusion que cette solution n'est pas si mauvaise.

Comme l'écrit pertinemment T. Liszcz ${ }^{7}$ : «La protection du salarié demeure la raison d'être essentielle du droit du travail, surtout dans le contexte actuel du capitalisme sauvage polonais et d'un taux de chômage toujours important. Remettre en question cette fonction équivaut, en substance, à s'interroger sur la raison d'être du droit du travail et à militer pour un retour vers l'Etat de droit du début du XIXe siècle, lorsque les relations du travail étaient régies par le droit civil avec ses principes standards : liberté contractuelle, égalité (juridique et formelle) des parties, non-ingérence de l'État dans les relations entre les parties au contrat».

La décision annulant le licenciement revêt un caractère constitutif ; en conséquence, toute la période non travaillée donne lieu à une rémunération. La décision prévoyant la réintégration n'a quant à elle pas le même caractère dans la mesure où il en découle un rétablissement du contrat de travail. L'employeur est chargé des coûts de la réintégration à hauteur du salaire pendant toute la durée non travaillée, sans dépasser néanmoins 2 mois, et pas plus d'1 mois dans le cas d'une période de préavis de 3 mois.

Les seules exceptions concernent les salariés ayant l'âge de la pré-retraite, les salariées durant la période de grossesse ou de congé de maternité, les pères élevant leurs enfants ou petits-enfants - ou d'autres membres de la famille la plus proche - pendant la période de congé de maternité ou lorsque la rupture du contrat de travail est soumise à certaines restrictions en vertu d'une disposition spécifique.

Dans ce cas, ces groupes bénéficient d'une rémunération pendant toute la période non travaillée. Toutefois, on ne peut pas considérer tous ces coûts comme des pertes supplémentaires susceptibles d'être supportées en vertu de l'application de l'art. $477 \S 2$ c.p.c. Si le tribunal de seconde instance ne modifie pas le jugement de première instance, alors les coûts seront les mêmes, mais il conviendra de les payer plus tard.

Dans ce contexte se pose cependant la question de savoir ce qu'il advient si le tribunal de seconde instance annule celui rendu par le tribunal de première instance et si l'employeur s'est vu imposer le maintien du contrat de travail jusqu'au terme définitif de la procédure. En effet, l'employeur devrait dans ce cas réclamer au Trésor Public des dommages et intérêts.

6 T. Liszcz, Droit du travail, 13e édition,Warszawa, 2019.

7 Ibid. 


\section{POLOGNE}

En dépit du fait qu'il semble légitime de considérer que la réforme charge les employeurs de façon excessive, cela peut les conduire à mieux réfléchir la décision de résilier un contrat de travail. La décision de réintégration ne prend pas effet automatiquement et reste soumise à l'exécution de mesures incontournables.

A ce jour, la doctrine attend la première décision de justice qui s'appuiera sur la disposition examinée dans le présent article afin d'évaluer son application. 